\title{
Laura SLATER, Art and Political Thought in Medieval
}

England, c. 1150-1350

Aude Mairey

\section{(2) OpenEdition}

\section{Journals}

Édition électronique

URL : https://journals.openedition.org/ccm/8167

DOI : $10.4000 / \mathrm{ccm} .8167$

ISSN : 2119-1026

\section{Éditeur}

Centre d'études supérieures de civilisation médiévale/Université de Poitiers

\section{Édition imprimée}

Date de publication : 1 septembre 2021

Pagination : 289-292

ISBN : 978-2-490783-10-6

ISSN : 0007-9731

Référence électronique

Aude Mairey, "Laura sLater, Art and Political Thought in Medieval England, c. 1150-1350 », Cahiers de civilisation médiévale [En ligne], 255 | 2021, mis en ligne le 01 septembre 2021, consulté le 02 février 2022. URL : http://journals.openedition.org/ccm/8167 ; DOI : https://doi.org/10.4000/ccm.8167

\section{(c) $(1)$}

La revue Cahiers de civilisation médiévale est mise à disposition selon les termes de la Licence Creative Commons Attribution - Pas d'Utilisation Commerciale - Pas de Modification 4.0 International. 
Laura Slater, Art and Political Thought in Medieval England, c. 1150-1350, Woodbridge, The Boydell Press (Boydell Studies in Medieval Art and Architecture), 2018.

L'ouvrage dense de Laura Slater porte sur l'imaginaire politique anglais entre le milieu du XII et le milieu du $\mathrm{XIV}^{\mathrm{e}} \mathrm{s}$. Plus précisément, il est consacré à la culture visuelle et à la manière dont cette dernière, en interaction avec les idées politiques de la période, a pu jouer un rôle pour façonner ou accompagner ces idées, dans le cadre de la culture politique qu'elle définit, à la suite de Peter Burke, comme les « croyances et pratiques par lesquelles l'autorité politique et les aspirations sont exprimées, pratiquées et débattues » (p. 6). Sur le plan historiographique, l'a. s'appuie notamment sur les récents travaux sur cette culture politique anglaise, tels ceux de John Watts, sur les conceptions de l'école de Cambridge (pour l'essentiel de John Pocock) ou, de manière plus diffuse, sur l'école des Annales.
L'a. définit ainsi sa démarche : «Je discute en premier lieu les penseurs politiques clés, les débats et les événements durant une période donnée. Je souligne ensuite des contributions visuelles spécifiques à ces arguments et les "acteurs-objets" placés de manière utile dans ces contextes » (p. 8). En ce qui concerne la définition d'une " image », l'a. s'appuie notamment sur les travaux classiques de Mary Carruthers et sur sa notion d' « image-mémoire » permettant de rendre visible des concepts abstraits.

Mais si elle entend mettre en relation les métaphores et les images, elle insiste sur la nécessité d'éviter un risque majeur, celui d'une équivalence stricte entre les deux. La métaphore du corps politique très prégnante au Moyen Âge par exemple, n'a pas vraiment généré d'équivalent visuel. Néanmoins, au 
cœur de son argumentation, se situe l'idée que « l'art et l'architecture révèlent beaucoup [de choses] sur les paramètres conceptuels au sein desquels les acteurs, les événements et les institutions politiques étaient situés par les contemporains ».

Dans ce cadre, ils participent selon l'a. d'une vision sacralisée de la monarchie anglaise, élaborée dans la seconde moitié du $\mathrm{XII}^{\mathrm{e}} \mathrm{s}$. et particulièrement

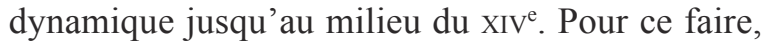
elle analyse tout au long de son livre quelques motifs récurrents - la mort des rois en lien avec le problème de la tyrannie, le thème de la floraison de la nature, le bouclier de la foi, etc. -, mais constamment réinterprétés, au sein d'un vaste corpus textuel et iconographique qu'elle qualifie elle-même d'arbitraire étant donné la foison de la matière (il ne sera d'ailleurs pas possible dans le cadre de ce compte rendu d'égrainer l'ensemble de ses sources).

Le premier chapitre est consacré à l'affirmation de cette vision sacralisée par un groupe de clercs, généralement formés dans les écoles parisiennes, proches de la cour (les curiales). L. Slater évoque notamment Jean de Salisbury, Richard FitzNigel, Pierre de Blois ou encore Giraud de Galles. Elle insiste sur plusieurs aspects de leurs conceptions politiques, enracinées dans leur formation théologique : la primauté de l'Église sur l'État, le rôle de guide du clergé et la piété du prince. Mais ces auteurs ont également des attaches avec les institutions ecclésiastiques de Canterbury, Christ Church en particulier, foyer intellectuel majeur, ce qui se reflète aussi dans la construction de la cathédrale après son incendie en 1174 .

L'a. s'arrête particulièrement sur l'œuvre de Jean de Salisbury (v. 1115-1180), à commencer par son Policraticus, et sur l'accent mis sur le thème de la tyrannie - qui parcourt l'ensemble de la période traitée dans l'ouvrage - en lien, ici, avec un augustianisme politique pessimiste et avec une vision selon laquelle les invasions et les guerres anglaises seraient liées avant tout aux péchés du peuple anglais. Mais Jean de Salisbury brode aussi sur un autre motif majeur, celui de la nature, de sa croissance et de sa floraison.

L. Slater s'attache ensuite, en contrepoint, au programme iconographique des vitraux de la cathédrale de Canterbury élaboré dans la seconde moitié des années 1170 (dont il ne nous reste que des fragments). Elle évoque en particulier le sixième vitrail typologique qui représente la parabole du semeur (Matthieu $13: 3-8$; Marc $4: 3-9$ ). On y retrouve l'importance de la nature et de sa croissance mais aussi son inverse, la terre dévastée, en lien avec deux personnages incarnant la tyrannie, les empereurs Julien l'Apostat et Maurice Tiberius, des hommes « épineux » en écho au livre des Juges (9:8-15). Selon l'a., ce vitrail constitue une clé « pour l'exploration par Jean de Salisbury de la conduite chrétienne positive et d'une harmonie politique "productive" dans le Policraticus » (p. 34). L'a. met également ce motif de la nature foisonnante avec le premier grand sceau de Richard Cœur de Lion, probablement réalisé v. 1189 et davantage susceptible, comme c'est le cas pour les sceaux en général, de toucher une large audience.

Un deuxième motif majeur est celui du piétinement des ennemis - tyrans compris - qui serait apparu dès le $\mathrm{IX}^{\mathrm{e}} \mathrm{s}$. dans le Psautier dit d'Utrecht, présent à Canterbury depuis le début du XI $\mathrm{e}$ s. À l'origine, c'est la Trinité qui piétine deux êtres divins, en rapport avec le premier verset du psaume 109. Cette image se retrouve dans plusieurs psautiers composés à Canterbury au XII ${ }^{e}$ s., mais elle est progressivement adaptée pour inclure rois couronnés et pécheurs ou, en regard du psaume $90: 13$, un lion et un dragon. Le motif est également repris dans des sceaux monastiques au tournant du XII ${ }^{e}$ et du XIII ${ }^{e}$ s., captant là encore un public plus large.

Dans la dernière partie de ce chapitre, l'a. se penche sur le cas de Thomas Becket (1120-1170), assassiné sur l'ordre d'Henri II, et dont le culte a été fondamental dans la vie politique anglaise. Or, dans les premières enluminures évoquant son martyr, T. Becket apparaît avant tout comme un exemple de bon gouvernement. Ainsi se met en place une vision sacralisée du gouvernement et de la monarchie qui irriguera les réflexions postérieures.

Le deuxième chapitre est consacré aux premières décennies du XIII ${ }^{\mathrm{e}}$ s., plus particulièrement à la guerre civile de 1215-1217 et à la minorité d'Henri III. Selon l'a., « la lutte politique était caractérisée en Angleterre par la guerre sacrée et entourée par des exhortations actives à la croisade, à la rébellion et à la résistance », et l'on observe de ce fait tant une évolution dans l'interprétation des motifs iconographiques que l'apparition de nouveaux éléments. 
Un point est tout d'abord effectué sur les idées de grands acteurs de l'époque, à commencer par Étienne Langton (v. 1150-1258), dont certaines conceptions font écho à l'époque précédente, particulièrement en ce qui concerne la primauté de l'Église, mais aussi sur la nécessité du conseil et de la correction, par exemple. À la suite de Philippe Buc, l'a. insiste sur l'imprégnation du lexique réformateur par le langage scolastique.

Elle évoque ensuite la généralisation d'un nouveau motif, celui des " pierres vivantes » que sont Noé, Daniel et Job (d'après Ézéchiel $14: 14$ ), appliqué aux grands évêques réformateurs de la période (É. Langton en premier lieu) incarnant la défense des opprimés - en l'occurrence le peuple anglais. Un autre motif important est celui de l'armure de Dieu, lié à la croisade dont les résonnances sont fortes dans le cadre de la vie politique anglaise de la période. Mais le scutum fidei, qui apparaît en premier lieu chez le chroniqueur Matthieu Paris, peut aussi bien être appliqué aux barons rebelles qu'aux partisans de la royauté.

Puis, l'a. analyse les développements des motifs exposés au chapitre précédent, celui de la nature foisonnante et celui du piétinement, qui apparaissent notamment dans le premier grand sceau d'Henri III, permettant ainsi leur large diffusion. En ce qui concerne les « pierres vivantes » et leurs extensions architecturales, elles apparaissent dans un autre sceau, celui de Londres (v. 1215-1219). L'importance de T. Becket est ensuite réaffirmée et la vision des saints royaux majeurs que sont St Edmond (m. 869) et St Édouard le confesseur (m. 1066) est analysée. Selon L. Slater, il existe une association entre ces trois saints, symboles d'un «pouvoir public» sanctifié.

Enfin, l'a. s'attache à la constitution de mythes entourant la Magna Carta en lien, notamment, avec É. Langton considéré comme son «père spirituel» mais aussi avec la bureaucratisation de l'État et l'importance de la loi et du droit, qui sont associées à la recherche du salut. Plus généralement, elle affirme que « dans les années 1220 , si ce n'est avant, une signification décisive sacrée et un objectif spirituel, si ce n'est croisé, a été donnée aux problèmes politiques de $1215 »$. La vision du pouvoir exprimée par les clercs du XII ${ }^{\mathrm{e}} \mathrm{s}$. est ainsi transformée pour répondre aux nécessités politiques du temps.
Le troisième chapitre porte principalement sur la guerre des barons de 1258-1263 et aux transformations intervenues dans ce contexte. L'a. analyse en premier lieu la pensée de Robert Grosseteste (v. 1175-1253), grand inspirateur de Simon de Montfort, leader du mouvement des barons. Si R. Grosseteste insiste tout autant que ses prédécesseurs sur la primauté de l'Église - en mettant peut-être davantage encore la nécessité de sa réforme - il est un des grands promoteurs de la pensée politique aristotélicienne (rappelons que c'est lui qui a traduit pour la première fois l'Éthique, entre autres). Celle-ci est caractérisée notamment par les notions de raison, de prudence et d'équité, que l'évêque de Lincoln articule avec la morale chrétienne. Ces préoccupations se retrouvent dans la littérature pastorale contemporaine, par exemple le Colloquium de Jean de Galles (v. 1265-1275). Ces idées auraient directement influencé le programme des réformateurs et dans ce cadre, Simon de Montfort apparaît également comme une " pierre vivante ».

L'a. analyse ensuite une série de manuscrits illustrés de l'Apocalypse, à commencer par la Lambeth Apocalypse (Londres, Lambeth Palace Library, MS 209, v. 1260-1275), dans lesquels on retrouve plusieurs motifs déjà évoqués, tels le bouclier de la foi ou la chute des mauvais empereurs, en particulier Julien l'Apostat. Toutefois, les destinataires de ces ouvrages sont difficiles à identifier et les références politiques peuvent tout aussi bien pencher du côté réformateur que du côté royaliste; mais cela induit, et c'est tout aussi important, l'existence d'un stock commun de références visuelles.

Mais dans d'autres œuvres clairement « royalistes », Simon de Montfort est vilipendé, car il est justement associé par l'intermédiaire de son héraldique aux tyrans déchus. Le thème de la mort des tyrans apparaît aussi dans la Painted Chamber de Westminster, refaite après un incendie en 1263, rappelant ainsi au roi et à ses proches les dangers d'un mauvais gouvernement. L'a. conclut le chapitre en notant que « durant la période de la guerre des barons, la réforme politique a continué à être vue comme une action dévotionnelle, étroitement connectée à, et équivalent dans son importance sacrée, à la réforme de l'Église et au soin pastoral pour le salut des âmes » (p. 162).

Le dernier chapitre analyse les transformations profondes ayant pris place entre la fin du XIII ${ }^{\mathrm{e}} \mathrm{s}$. et le milieu du XIV ${ }^{\mathrm{e}}$, même s'il existe des continuités. Les saints politiques par exemple (Édouard, 
Edmond, Becket, rejoints au début du XIV ${ }^{\mathrm{e}}$ par Thomas de Lancastre) ont toujours un rôle important dans la dimension salvatrice de la monarchie; en corollaire, la tyrannie est toujours une préoccupation majeure.

L'a. analyse tout d'abord, dans les principales chroniques de la période, l'émergence d'une nouvelle vision de l'histoire délaissant le modèle du peuple pécheur pour embrasser une continuité historique des différentes dynasties. Elle évoque ensuite les rouleaux généalogiques qui se développent à la fin $\mathrm{du} \mathrm{XIII}^{\mathrm{e}} \mathrm{s}$. et qui constituent des « histoires vivantes »; elles mettent l'accent, encore et toujours, sur la mort des rois et des tyrans. Cette vision apparaît également dans certaines chroniques abrégées en prose, telle celle du manuscrit de Londres, le Cotton Vittelius A XIII.

L. Slater étudie ensuite le traité de Walter de Milemete, probablement un clerc royal, sans doute composé pour le jeune Édouard (III) au moment de la crise politique ayant mené à la déposition de son père, en 1326-1327 donc. Ce traité, abondamment illustré, suggère une importance nouvelle placée sur l'éducation du prince tout en révélant une anxiété profonde sur la situation contemporaine. L'a. s'attache ensuite aux changements iconographiques intervenus dans les grands sceaux royaux, en particulier l'abandon du motif du piétinement en 1340. Elle en conclut à la diminution de la force des images par rapport à la « lutte sacrée ».

Cela est lié, selon elle, à des transformations radicales causées par trois facteurs principaux : la multiplication des conflits militaires qui a pour conséquence d'élargir la société politique, mais aussi de déplacer les critiques vers le nerf de la guerre - les taxes, en argent et en nature; la redéfinition des liens féodaux avec une insistance sur la gloire chevaleresque; et la croissance de la bureaucratie et des institutions, en particulier du Parlement, qui indique un changement de nature du « public politique ». L'a. conclut que, malgré la persistance de nombreux motifs, même transformés, un véritable tournant prend place au milieu du $\mathrm{XIV}^{\mathrm{e}} \mathrm{s}$., en matière de réception des idées politiques et de leur visualisation.

L'ouvrage de L. Slater est dense, voire touffu et l'on se perd parfois dans l'abondance des exemples et l'intrication de l'histoire des différents motifs évoqués tout au long des quatre chapitres. Cette impression est renforcée par le fait que le contexte politique précis n'est souvent qu'esquissé (sauf dans le dernier chapitre), ce qui nécessite, pour tirer profit au mieux de cette étude, de bien connaître l'histoire politique anglaise. Enfin, malgré l'abondance des reproductions, certaines descriptions d'œuvres importantes n'en sont pas accompagnées - c'est le cas, par exemple, des rouleaux généalogiques. Cela dit, si l'a. reconnaît en conclusion que son étude est forcément partielle, son analyse de l'iconographie de différents types de documents, qu'il s'agisse de peintures murales, de tombes, de sceaux ou encore de livres dévotionnels, en lien avec la rhétorique politique de l'époque, offre une approche fort stimulante.

Aude MaIrEY

UMR 8589 - Lamop/CNRS - Paris 1 